$1 / 19 / 94$

\title{
DIFFUSION-LIMITED AGGREGATION AS BRANCHED GROWTH
}

\author{
Thomas C. Halsey \\ The James Franck Institute and Department of Physics \\ The University of Chicago \\ 5640 South Ellis Avenue \\ Chicago, Illinois 60637
}

\begin{abstract}
I present a first-principles theory of diffusion-limited aggregation in two dimensions. A renormalized mean-field approximation gives the form of the unstable manifold for branch competition, following the method of Halsey and Leibig [Phys. Rev. A 46, 7793 (1992)]. This leads to a result for the cluster dimensionality, $D \approx 1.66$, which is close to numerically obtained values. In addition, the multifractal exponent $\tau(3)=D$ in this theory, in agreement with a proposed "electrostatic" scaling law.
\end{abstract}

PACS Numbers: 64.60A, 68.70, 05.20 
Diffusion-limited aggregation (DLA) is a model of pattern formation in which clusters grow by the accretion of successive random walkers. ${ }^{1}$ Each random walker arrives from infinity, and sticks to the growing cluster at whichever surface point it first contacts. Only after the accretion of a walker does the next walker commence its approach to the cluster. The clusters thereby obtained are fractal in all dimensionalities $d>1$, and are qualitatively and/or quantitatively similar to patterns observed in such diverse phenomena as colloidal aggregation, electrodeposition, viscous fingering, and dielectric breakdown. ${ }^{2}$

At the heart of the problem of diffusion-limited aggregation is the following question: what is the relationship between the scale-invariance of the diffusive growth process and the hierarchical structure of the clusters generated by this process? ${ }^{3}$ A preliminary, and incomplete, answer to this question was provided by this author in collaboration with M. Leibig. ${ }^{4}$ In this work, it was hypothesized that the quantitative process by which one branch screens, i.e., takes growth probability from, a neighboring branch, has a specific form, independent of the length scale on which this process takes place. This assumption allows the development of a qualitatively correct theory, which yields multifractal scaling of growth probability, as well as agreement with a phenomonological scaling law, the "Turkevich-Scher" law, relating the scaling of the maximum growth probability over all sites on the cluster to the dimension of the cluster as a whole. ${ }^{5}$

In this letter, I shall present a more complete and a priori theory of diffusionlimited aggregation in two dimensions based upon a specific mean-field calculation of the dynamics of branch competition. Because the mean-field approximation is implemented on all length scales, it is perhaps better to regard this theory as an ansatz solution in the case where certain types of fluctuations on all length scales are neglected, while others are included. This specific model allows verification of 
all qualitative aspects of branch competition that were advanced as (reasonable) hypotheses in Ref. 4. The result obtained for the dimensionality of the cluster, $D=1.66$, is within $3 \%$ of the oft-quoted value $D=1.71$ obtained from the scaling of the cluster radius-of-gyration in numerical studies. An additional scaling law (the "electrostatic scaling law"), relating the multifractal exponent $\tau(3)$ of the growth measure to the dimensionality $D$ by $D=\tau(3)$, is seen to be exact within this theory. ${ }^{6}$

In the growth process, each particle attaches itself to a unique "parent" particle in the pre-existing cluster. Furthermore, the cluster is observed to be a branched structure, with no loops and with each particle having asymptotically zero, one or two "children", i.e. particles to whom it stands as a parent. ${ }^{7}$ Very rarely particles have more than two children; primarily for reasons of convenience I neglect this possibility.

Consider a particle with two children. Each of the two children separately, with all particles descended from each, I term a "branch". Thus these two-child particles are parents of two branches, which occupy neighboring regions of space. The total number of particles in one branch I term $n_{1}$, and the total in the other $n_{2}$. The total number of descendants of the parent particle is thus $n_{b} \equiv n_{1}+n_{2}$. Now consider the next particle to accrete to the cluster. I say that this particle has a total probability $p_{1}$ to stick anywhere on the first branch, and a total probability $p_{2}$ to stick anywhere on the second branch, yielding a total probability $p_{b} \equiv p_{1}+p_{2}$.

Let us now consider the normalized quantities $x=p_{1} / p_{b}$ and $y=n_{1} / n_{b}$. Clearly $d n_{1} / d n=p_{1}$, where $n$ is the total number of particles in the cluster, and we are neglecting fluctuations of $O\left(\sqrt{n}_{b}\right)$. Thus $y$ obeys the following equation of motion: 


$$
\frac{d y}{d \ln n_{b}}=x-y
$$

The right-hand side of this equation is a function only of $x$ and $y$. Now $x$ will obey an equation of the form

$$
\frac{d x}{d \ln n_{b}}=G\left(x, y ; n ;\left\{\phi_{i}\right\}\right),
$$

where $\left\{\phi_{i}\right\}$ is some parameterization of all of the variables describing the structure of the cluster. In ref. 4 we assumed that by averaging the right-hand side of this equation over these parameters $\left\{\phi_{i}\right\}$, one obtains $d x / d \ln n_{b}=g(x, y)$, where the right-hand side is now only a function of $x$ and $y$. Given this function $g(x, y)$, one has a closed system of equations describing the evolution of $x$ and $y$ as functions of $\ln n_{b}$

By symmetry, $g(x, y)=-g(1-x, 1-y)$, so $(x, y)=(1 / 2,1 / 2)$ must be a fixed point of this process of competition between the two branches. In ref. 4, we explored the consequences of assuming that this fixed point is hyperbolic, with the unstable manifold emerging from the fixed point terminating in two stable fixed points at $(x, y)=(0,0)$ or $(x, y)=(1,1)$, these latter representing the situation in which one branch has been completely screened by the other. This assumption will be explicitly verified in the calculation below.

If the central fixed point at $(x, y)=(1 / 2,1 / 2)$ is hyperbolic, then branch pairs which commence their existence (with $n_{b} \sim 1$ ) near the unstable fixed point will be quickly drawn onto the unstable manifold. Linearizing the system of equations for $d(x, y) / d \ln n_{b}$ about the central fixed point, the hyperbolic assumption implies that there will be a stable and an unstable direction; the eigenvalue corresponding to the latter direction we define to be $\nu$. 
When a pair of branches is first created by a tip-splitting event, its initial growth up to the stage at which $n_{b} \gg 1$ is determined by complicated microscopic dynamics, which do not recognize the existence of the unstable fixed point. Thus we expect the probability that a newly created branch pair will be a distance $\epsilon^{\nu}$ from the unstable fixed point will be $\rho(\epsilon \ll 1) d \epsilon \propto \epsilon^{\nu-1} d \epsilon$; we are assuming a constant probability density of branch creation near the unstable fixed point. This assumption has been specifically verified by numerical study in ref. 4. The choice of $\epsilon^{\nu}$ for this initial distance insures that position along the unstable manifold in the $x-y$ plane can be parameterized by the variable $\epsilon n_{b}$.

It is possible to relate the eigenvalue $\nu$ to the cluster dimensionality $D$ by the following argument. ${ }^{4}$ Consider the strongest branch in the cluster, that obtained by always following the stronger child (with the larger values of $x, y$ ) at each branching. The total number of side-branches (or branch points) from such a branch is $\sim r$, where $r$ is the cluster radius. In order that the cluster have a dimension $D>1$, a number $\sim 1$ of these side branches must have a total number of particles $\sim n$, the total number in the cluster. A side branch obeying this criterion must have $\epsilon n \sim 1$, so that at that branching, both descendant branches are roughly equal in size. The probability of this happening at any particular branching is $\int^{n^{-1}} d \epsilon \rho(\epsilon) \propto n^{\nu}$, and there are $\sim r$ different sidebranchings at which this might occur. Thus $r n^{\nu} \sim 1$, or $D=1 / \nu$

In order to determine $g(x, y)$, we turn to an explicit description of the growth process. ${ }^{6,8}$ Suppose that we parameterize the accessible surface of the cluster by arc-length $s$. If a particle attaches at the surface point $s^{\prime}$, it thereby reduces the growth probability at all points $s$ for which $\left|s-s^{\prime}\right|>a$, where $a$ is the particle size. This is because a certain number of the random walks that would have reached $s$ previously are now obstructed by the new particle at $s^{\prime}$. If the probability that a 
particle lands at $s^{\prime}$ is $p\left(s^{\prime}\right)$, and the probability that a random walker goes from $s^{\prime}$ to $s$ without contacting the surface is $H\left(s, s^{\prime}\right)$, this implies that

$$
\frac{d p(s)}{d n}=\int d s^{\prime}\left(H\left(s, s^{\prime}\right)-h(s) \delta\left(s-s^{\prime}\right)\right) p^{2}\left(s^{\prime}\right),
$$

where we have modelled effects on the scale $\left|s-s^{\prime}\right|<a$ by the $\delta$-function, the coefficient of which, $h(s)$, is set by the conservation of the total growth probability, $\int d s p(s)=1$. Note that in Eq. (3), two factors of $p\left(s^{\prime}\right)$ appear-one corresponds to the original probability that a particle lands at $p\left(s^{\prime}\right)$, the other to the potential trajectories arriving at $s$ that are blocked by such a particle.

For $a \ll\left|s-s^{\prime}\right| \ll a n$, conformal transformation shows that the function $H\left(s, s^{\prime}\right)$ is given in two dimensions by the simple form ${ }^{9}$

$$
H\left(s, s^{\prime}\right)=\frac{p(s) p\left(s^{\prime}\right)}{\left[\int_{s}^{s^{\prime}} d s^{\prime \prime} p\left(s^{\prime \prime}\right)\right]^{2}},
$$

where the integral in the denominator is the total growth probability between the points $s$ and $s^{\prime}$. It is convenient to parameterize the interface by this quantity, the "growth probability" distance between points $z(s)$, defined by $z\left(s^{\prime}\right)-z(s)=$ $\int_{s}^{s^{\prime}} d s^{\prime \prime} p\left(s^{\prime \prime}\right)$. Then our fundamental equation becomes

$$
\frac{d p(z)}{d n}=p(z) \int d z^{\prime}\left[\frac{1}{\left(z-z^{\prime}\right)^{2}}-\tilde{h}(z) \delta\left(z-z^{\prime}\right)\right] p^{2}\left(z^{\prime}\right),
$$

where $a$ serves as an ultra-violet cutoff to prevent divergence of the integral, and $\tilde{h}(z)$ is related to $h(s)$ and to the function $z(s)$; its precise form is of no interest to us.

I wish to use this equation to determine the function $d x / d \ln n_{b}=g(x, y)$. Repeated application of the chain rule yields 


$$
\frac{d x}{d \ln n_{b}}=\frac{n_{b}}{p_{b}^{2}}\left\{(1-x) \frac{d p_{1}}{d n}-x \frac{d p_{2}}{d n}\right\}
$$

Consider a branch with probability $p^{\prime}$ and a number of particles $n^{\prime}$. We suppose that this branch extends from $z=0$ to $z=p^{\prime}$. Eqs. (5) and (6) imply that if we can write $p^{2}(z)$ on this branch (and by extension, all other branches) as

$$
p^{2}(z)=\frac{\left(p^{\prime}\right)^{2}}{n^{\prime}} f\left(z / p^{\prime}\right)
$$

where $f(z)$ is a universal function that depends neither upon $p^{\prime}$ nor upon $n^{\prime}$, then we will be able to write $d x / d \ln n_{b}=g(x, y)$, with the right-hand side a function of $x$ and $y$ alone. Equation (7) is motivated by the fact that $p^{2}(z)$ must be proportional to $\left(p^{\prime}\right)^{2}$; the dependence on $n^{\prime}$ is specifically chosen to lead to an $n^{\prime}$-independent $g(x, y)$. Only if we can find a method of computing an $n^{\prime}$-independent $f(z)$ will this ansatz be justified.

Thus the crux of the problem is this "branch envelope" function $f(z)$, which represents, with the appropriate normalization, the distribution of growth probability in different regions of a branch. Now in our picture, each branch can be divided into two distinct sub-branches, which compete according to the dynamics established by $g(x, y)$. Our central mean-field assumption is that we can compute $f(z)$ by averaging the envelope functions $f(z)$ of these sub-branches over the stochastic parameter $\epsilon$ appropriate to the competition of these two sub-branches. In this way we obtain the following equation:

$$
f(z)=\int_{-\infty}^{\infty} d \epsilon \rho(\epsilon)\left\{\frac{x^{2}\left(\epsilon n_{b}\right)}{y\left(\epsilon n_{b}\right)} f\left(\frac{z}{x\left(\epsilon n_{b}\right)}\right)+\frac{\left(1-x\left(\epsilon n_{b}\right)\right)^{2}}{\left(1-y\left(\epsilon n_{b}\right)\right)} f\left(\frac{1-z}{1-x\left(\epsilon n_{b}\right)}\right)\right\}
$$


where $x\left(\epsilon n_{b}\right)$ and $y\left(\epsilon n_{b}\right)$ give the values of $x$ and $y$ along the unstable manifold as functions of $n_{b}$ and the stochastic parameter $\epsilon$. For convenience, we are defining $\rho(\epsilon)$ for negative values of $\epsilon$ as $\rho(-\epsilon)=\rho(\epsilon)$, with $x(-\eta)=1-x(\eta), y(-\eta)=1-y(\eta)$. This leads to the relatively compact expression of Eq. (8). For large $n_{b}$, this equation has a solution independent of $n_{b}$, which is determined by

$$
\int_{-\infty}^{\infty} d \eta|\eta|^{\nu-1}\left\{\frac{x^{2}(\eta)}{y(\eta)} f\left(\frac{z}{x(\eta)}\right)+\frac{(1-x(\eta))^{2}}{(1-y(\eta))} f\left(\frac{1-z}{1-x(\eta)}\right)-f(z)\right\}=0
$$

Since the integrand goes to zero as $\eta \rightarrow \infty$, we are justified in taking the small $\epsilon$ form for $\rho(\epsilon)$.

Of course, in order to perform this integral, we must have the form of the unstable manifold, and thus we must already know $g(x, y)$. We can determine $g(x, y)$ from $f(z)$ by simply integrating Eq. (5) over the appropriate intervals. We do not integrate over regions exterior to the two competing branches, but only investigate the influence of the two branches on one another. Skipping some tedious algebra, we may express the result as follows. Defining a function $\psi(u)$ by

$$
\psi(u)=\int_{0}^{1} d z\left(\frac{1}{z}-\frac{1}{z+u}\right) f(z)
$$

we can write

$$
\begin{aligned}
g(x, y)= & x(1-x)\left\{\left[2 \frac{x}{y} \psi(\infty)-\frac{(1-x)^{2}}{(1-y) x} \psi\left(\frac{x}{1-x}\right)\right]\right. \\
& \left.-\left[2 \frac{1-x}{1-y} \psi(\infty)-\frac{x^{2}}{y(1-x)} \psi\left(\frac{1-x}{x}\right)\right]\right\} .
\end{aligned}
$$

The reader should note that we have a circular procedure, because $g(x, y)$ is determined as a function of $f(z)$ by Eqs. (10) and (11), while $f(z)$ is determined as 
a function of $g(x, y)$, and in particular by the unstable manifold in the $x-y$ plane as determined by $g(x, y)$, by Eq. (9). Thus in practice we are looking for a solution of Eq. (9) where the functions $x(\eta)$ and $y(\eta)$ are implicitly determined by $f(z)$.

I have numerically obtained the unique solution to Eq. (9) under these conditions, which is displayed in the inset to Figure $1 .{ }^{10}$ This validates our assumption regarding the scaling with $n^{\prime}$ in Eq. (7). The function $g(x, y)$ determined from this function has all of the necessary qualitative features; in particular, the fixed point at $(x, y)=(1 / 2,1 / 2)$ is unstable and hyperbolic, and the unstable manifold leads from this point to stable fixed points at $(x, y)=(0,0)$ and $(1,1)$, as illustrated in Figure 1. Figure 1 also shows numerical results for branch competition. The value of the unstable eigenvalue $\nu$ is $\nu \approx .6020$, implying that $D=1 / \nu \approx 1.661$, which is within $3 \%$ of the standard numerical result $D \approx 1.71$.

In addition, this theory automatically agrees with the electrostatic scaling law, which states that

$$
\left\langle\int d s p(s)^{3}\right\rangle \propto n^{-1}
$$

where the integral is over the entire cluster surface. This is equivalent to the more usual statement that $\tau(3)=D$. In ref. 4 , we demonstrated that the multifractal exponents $\sigma(q)$ defined by $\int d s p(s)^{q} \propto n^{-\sigma(q)}$ can be obtained from the integral condition $^{11}$

$$
\int_{0}^{\infty} d \eta \eta^{\nu-1}\left\{\frac{x(\eta)^{q}}{y(\eta)^{\sigma(q)}}+\frac{(1-x(\eta))^{q}}{(1-y(\eta))^{\sigma(q)}}-1\right\}=0
$$

By integrating Eq. (9) from $z=0$ to $z=1$, one obtains precisely this criterion, with $q=3$ and $\sigma(q)=1$, in agreement with the electrostatic scaling law. Though the electrostatic scaling law thus appears in a natural way in this theory, one should 
not say that it is predicted by this theory unless the solution obtained to Eq. (9) is stable. It may be that it is necessary to impose the electrostatic scaling law as a constraint to insure this stability. ${ }^{10}$

From Figure 1, it is clear that although in some sense the unstable manifold that we have calculated is an acceptable average trajectory, the numerically obtained trajectories do exhibit some dispersion about this average. This has significant results. The Makarov scaling law predicts that $d \sigma(q) /\left.d q\right|_{q=1}=1 / D_{0},{ }^{12}$ where $D_{0}$ is the surface fractal dimension (which according to some studies is significantly less than the radius-of-gyration dimension $D.)^{13}$ My result, from Eq. (13), is $d \sigma(q) /\left.d q\right|_{q=1} \approx 0.71$, which is significantly different from the Makarov result. In practice, this quantity is quite sensitive to the way in which the unstable manifold approaches the stable fixed points at $(x, y)=(0,0)$ and $(1,1)$; since the numerical trajectories are quite dispersed in this region, I do not expect a good result for the Makarov scaling from a one-trajectory theory. However, the theory outlined in this letter can be easily generalized to account for the possibilty of trajectory dispersion, which may lead to better agreement with the Makarov result.

\section{Acknowledgements}

I would like to acknowledge a stimulating discussion with L.P. Kadanoff, as well as conversations with R. Blumenfeld on a closely related topic. I am very grateful to A. Libchaber for encouragement at an early stage in this project. This work was supported by the National Science Foundation through a Presidential Young Investigator award, Grant DMR-9057156. 


\section{Notes}

1. T.A. Witten, Jr. and L.M. Sander, Phys. Rev. Lett. 47, 1400 (1981); P. Meakin, Phys. Rev. A 27, 1495 (1983).

2. R. Brady and R.C. Ball, Nature (London) 309, 225 (1984); L. Niemeyer, L. Pietronero, and H.J. Wiesmann, Phys. Rev. Lett. 52, 1033 (1984); J. Nittmann, G. Daccord, and H.E. Stanley, Nature (London) 314, 141 (1985).

3. This question is also the focus of real-space studies such as L. Pietronero, A. Erzan, and C. Evertsz, Phys. Rev. Lett. 61, 861 (1988); Physica A 151, 207 (1988), and X.R. Wang, Y. Shapir and M. Rubenstein, Phys. Rev. A 39, 5974 (1989); J. Phys. A 22, L507 (1989).

4. T.C. Halsey and M. Leibig, Phys. Rev. A 46, 7793 (1992); T.C. Halsey and K. Honda, unpublished.

5. L. Turkevich and H. Scher, Phys. Rev. Lett. 55, 1026 (1985); Phys. Rev. A 33, 786 (1986); see also R. Ball, R. Brady, G. Rossi, and B.R. Thompson, Phys. Rev. Lett. 55, 1406 (1985), and T.C. Halsey, P. Meakin, and I. Procaccia, Phys. Rev. Lett. 56, 854 (1986).

6. T.C. Halsey, Phys. Rev. Lett. 59, 2067 (1987); Phys. Rev. A 38, 4749 (1988).

7. The fact that there are no loops follows from the fact that every particle has a unique parent, which is true in off-lattice versions of DLA.

8. B. Shraiman and D. Bensimon, Phys. Rev. A 30, 2840 (1984); R.C. Ball and M. Blunt, Phys. Rev. A 39, 3591 (1989).

9. T.C. Halsey, Phys. Rev. A 35, 3512 (1987).

10. The stability of this solution is a more difficult question. There appears numerically to be a single instability of the solution, which can be eliminated if one applies the electrostatic scaling law as a constraint. 
11. For a general discussion of multifractality, see T. Vicsek, Fractal Growth Phenomena, 2nd ed. (World Scientific, Singapore, 1992).

12. N.G. Makarov, Proc. London Math. Soc. 51, 369 (1985).

13. In particular, $D_{0} \approx 1.60$ is claimed by F. Argoul, A. Arneodo, G. Grasseau, and H. Swinney, Phys. Rev. Lett. 61, 2558 (1988). 


\section{Figure Caption}

1. Trajectories of branch competion in the $x-y$ plane. The light solid trajectories are numerical results from ref. 4 for specific branch pairs in growing DLA clusters. The heavy solid line represents the unstable manifold predicted by this letter, which is quite close to the "average" numerical trajectory. The inset shows the computed branch envelope function $f(z)$. 